\title{
Wetting and interfacial order at nematic free surfaces
}

\author{
E. Martín del Río $\left.{ }^{*}\right)$, M. M. Telo da Gama ${ }^{1}$, E. De Miguel ${ }^{1}\left(^{*}\right)$ and L. F. Ruld ${ }^{2}$ \\ 1 Departamento de Física da Faculdade de Ciências \\ Centro de Física da Matéria Condensada, Universidade de Lisboa \\ Av. Prof. Gama Pinto 2, 1699 Lisboa Codex, Portugal \\ 2 Departamento de Física Atómica, Molecular y Nuclear, Universidad de Sevilla \\ Apartado 1065, Sevilla 41080, Spain
}

(received 7 March 1996; accepted in final form 31 May 1996)

PACS. $61.30 \mathrm{Cz}$ - Theory and models of liquid crystals.

PACS. 68.45Gd - Wetting.

PACS. 68.10Cr - Surface energy (surface tension, interface tension, angle of contact, etc.).

\begin{abstract}
We apply a density-functional theory to the free surface of a model liquid crystal, to investigate the orientational order at the nematic free surface of systems exhibiting orientational wetting at the isotropic liquid-vapour interface. It is shown that the theory accounts for the growth of the surface ordered layer which develops at the nematic-vapour interface, as the temperature increases up to the triple point, as was recently reported for 4 -cyano- $4^{\prime}-n$-alkyl-biphenyls. We have found a correlation between the growth of this layer and the non-monotonic temperature dependence of the interfacial tension below the triple point. The theoretical results are in excellent agreement with the results for $8 \mathrm{CB}$.
\end{abstract}

Fluids of strongly anisotropic molecules, such as liquid crystals, exhibit a large variety of fluid phases, characterized by different types of long-range order (orientational and positional). The description of the fluid interfaces (density profiles and interfacial tension) between two (or more) of these phases is an extremely interesting problem both theoretically as well as regarding potential applications (e.g., displays). In the simplest case of planar interfaces the broken translational symmetry induces, in general, a lower rotational symmetry at the interface and thus surface-induced alignment is expected to be important when liquid crystalline phases are involved [1]. As a consequence, nematic free surfaces exhibit a wealth of behaviour characterized by a wide variety of interfacial orders and structural phase transitions (wetting, anchoring, etc.) may (and do) occur [1].

The first attempt to account for the existence of a preferred molecular orientation at the nematic-isotropic liquid (NI) interface was made by de Gennes [2]. Although several theories were developed following de Gennes' pioneering work, their consideration of a single orientational order parameter implies that there is no excess order at the nematic-vapour (NV) interface [3], by contrast with recent experimental observations.

Indeed, Kasten and Strobl [4] have carried out a detailed study of the free surface of the homologous series of 4-cyano-4'- $n$-alkyl-biphenyls $(n \mathrm{CB})$, with $n=5,6,7$, 8, by using reflection ellipsometry. They found that the isotropic-vapour (IV) interface is completely wet by a homeotropically aligned nematic $(\mathrm{N})$ film for $n=6,7,8$, while partial wetting ocurs for

$\left({ }^{*}\right)$ Permanent address: Departamento de Física Atómica, Molecular y Nuclear, Universidad de Sevilla, Apartado 1065, Sevilla 41080, Spain.

(C) Les Editions de Physique 
$n=5$. Of particular relevance, as will be discussed in this letter, is the fact that a strongly ordered surface layer was found to develop at the NV interface. This ordered surface layer grows for systems with $n=6,7,8$ as the temperature $(T)$ increases towards the triple-point temperature $\left(T_{\mathrm{tr}}\right)$. As far as we know, there are no theoretical descriptions of the growth of this layer.

More than a decade ago, one of us [5] developed a generalized van der Waals theory for non-uniform molecular fluids. This theory was applied to model nematic liquid crystals with anisotropic attractive interactions truncated at the Maier-Saupe level. No preferred orientation at the interface is obtained for this model. The theory was subsequently applied to a more realistic (although still highly idealized) model by Thurtell et al. [6] and Tjipto-Margo et al. [7], by including an extra term in the intermolecular potential coupling the translational and rotational degrees of freedom. This extended model yields different equilibrium tilt angles for prolate and oblate molecules.

More recently we concluded a detailed study of the orientational effects induced at the NI and NV interfaces of a slightly more general model liquid crystal [8]. One of the most important results of this study is the description of the experimental observation of different molecular alignments at the NV and NI interfaces of a given nematogen, as the result of a competition between different terms in the anisotropic intermolecular potential. We have, however, failed to predict the ordered surface layer observed recently by Kasten and Strobl in the nematic phase [4].

In the following, we offer a theoretical description of this effect and relate it to the nonmonotonic behaviour of the surface tension of the nematic-vapour interface below the triplepoint temperature [9]. We used the simplest (local) version of density-functional mean-field theory as described in previous work [8] and restricted the calculations to the simplest nontrivial model. In this model the molecules are assumed to be uniaxial with a spherically symmetric hard core (of diameter $\sigma$ ) and an attractive interaction accounting for the anisotropy of the intermolecular potential. Each molecule is represented by the position of its centre of mass, $\mathbf{r}$, and a unit vector $\mathbf{u}$ in the direction of its symmetry axis. The attractive potential is thus written as

$$
u_{\text {att }}\left(r_{i j}, \hat{\mathbf{r}}_{i j}, \mathbf{u}_{i}, \mathbf{u}_{j}\right)=-\epsilon\left(\frac{\sigma}{r_{i j}}\right)^{6}\left\{1+B P_{2}\left(\mathbf{u}_{i} \cdot \mathbf{u}_{j}\right)-C\left[P_{2}\left(\hat{\mathbf{r}}_{i j} \cdot \mathbf{u}_{i}\right)+P_{2}\left(\hat{\mathbf{r}}_{i j} \cdot \mathbf{u}_{j}\right)\right]\right\},
$$

where $\epsilon$ sets the energy scale, the term proportional to $B$ (Maier-Saupe) drives the bulk nematic transition and the last term (proportional to $C$ ) couples the rotational and translational degrees of freedom, yielding non-trivial surface behaviour. Within our approximate theory, the parameter $C$ has no influence on the bulk phase behaviour when translationally invariant phases are considered. We do not consider bulk smectic phases since they are not expected to be stable for models with spherical hard cores such as the one used in this work [10].

We have studied the interfacial properties of systems with $B=0.3$ (and different values of $C$ ) exhibiting three fluid phases (nematic, isotropic liquid (I), and vapour (V)) which coexist at a triple point $\left(T_{\mathrm{tr}}=0.2182\right.$, in units of $\epsilon / k_{\mathrm{B}}$, where $k_{\mathrm{B}}$ is Boltzmann's constant [8]). The phase diagram is sketched on the inset of fig. 1 . We recall that the equilibrium tilt angle is found to be $\Psi=0^{\circ}$ (homeotropic aligment) for $C>0$ (oblate molecules) and $\Psi=90^{\circ}$ (homogeneous planar alignment) for $C<0$ (prolate molecules) [6], [7].

The wetting behaviour of this model was studied by Tjipto-Margo et al. who found that firstorder wetting transitions of the IV interface by $\mathrm{N}$ occur when $C=0.8477$ and $C=-2.1249$. These authors also predict the non-monotonic behaviour of the surface tension $\gamma_{\text {IV }}$ above the triple point for systems exhibiting complete wetting by $\mathrm{N}$ [7]. The excess order at the NV interface, however, was overlooked in this and related studies [8] and the non-monotonic behaviour of the surface tension $\left(\gamma_{\mathrm{NV}}\right)$ below the triple point has not been obtained. 


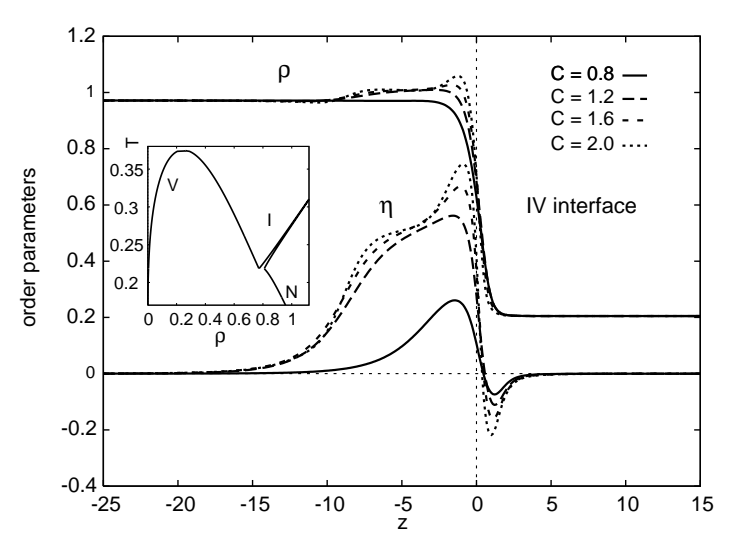

Fig. 1.

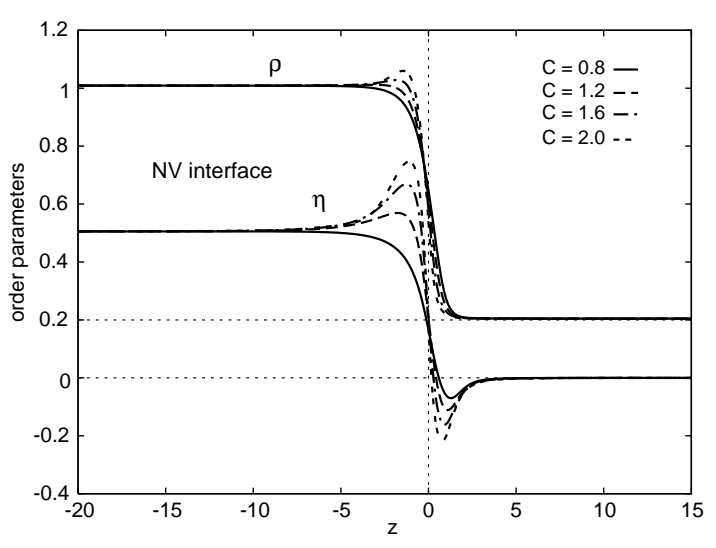

Fig. 2 .

Fig. 1. - Density and orientational order parameter profiles at the isotropic liquid-vapour interface for systems with $B=0.3$ and different values of $C$, at the scaled temperature $t=1.37 \times 10^{-3}$. For clarity, the density profile has been shifted upwards by 0.2 . The temperature-density phase diagram is shown in the inset. The density is in units of $\sigma^{3}, z$ in units of $\sigma$, and the temperature is in units of $\epsilon / k_{\mathrm{B}}$.

Fig. 2. - Density and orientational order parameter profiles at the nematic-vapour interface for systems with $B=0.3$ and different values of $C$, at the triple-point temperature. As $C$ increases, a strongly ordered surface layer develops at the interface. The density is in units of $\sigma^{3}$ and $z$ in units of $\sigma$. For clarity, the density profile has been shifted upwards by 0.2 .

Prompted by the striking experimental results of Kasten and Strobl [4] we decided to investigate the behaviour of the NV interface more systematically.

We have calculated the density and order parameter profiles, as well as the surface tension, $\gamma$, of the free surface of the model, at temperatures close to $T_{\mathrm{tr}}$, for values of $C$ in the range $0.8 \leq C \leq 2.0$. The density and order parameter profiles are plotted in fig. 1 (IV interface at $t=1.37 \times 10^{-3}$, where $\left.t=\left(T-T_{\mathrm{tr}}\right) / T_{\mathrm{tr}}\right)$ and in fig. $2\left(\mathrm{NV}\right.$ interface at $\left.T=T_{\mathrm{tr}}\right)$ for systems characterized by different values of $C$. Since the interface is uniaxial, a single orientational order parameter, $\eta$, is required, in addition to the density $\rho$, to describe the interfacial structure. This order parameter is defined as $\eta(z)=\left\langle P_{2}(\cos \theta(z))\right\rangle$, where the angular brackets denote a statistical average and $\theta(z)$ is the angle between the axial vector of one molecule and the $z$-axis (perpendicular to the interface). The results clearly indicate that the interfacial order increases with $C$, in agreement with previous work. Of particular relevance, however, is the fact that for the two largest values of $C$, the excess interfacial order is coupled to a variation in the density profile: a thin "smectic-like" layer develops at the NV interface, as indicated by the non-monotonic behaviour of $\rho(z)$. A similar effect is observed at the IV interface. As will be discussed later, this anomalous surface order is closely related to the anomalous behaviour of $\gamma_{\mathrm{NV}}$ close to $T_{\mathrm{tr}}$.

We define the nematic coverage, $\Gamma$, as $\Gamma=\int_{-\infty}^{0}\left[\eta(z)-\eta_{\text {bulk }}\right] \mathrm{d} z$, where $\eta_{\text {bulk }}$ is the nematic order parameter in the bulk phase (i.e. $\eta_{\mathrm{bulk}}=0$ for the IV interface). The nematic coverage is related to the zeroth moment of the anisotropic dielectric surface profile, $\Delta \varepsilon(z)$, measured by reflection ellipsometry [4]. The behaviour of $\Gamma$ close to the triple point $(t \rightarrow 0)$ is shown in fig. 3. The order of magnitude of our data agrees with the experimental results if we take $\sigma=10 \AA$, which is a reasonable value for the $n \mathrm{CB}$ molecules (see fig. $4 a$ ) in [4]).

If the system exhibits complete wetting by $N$ (for $C>0.8), \gamma_{\mathrm{IV}}=\gamma_{\mathrm{NI}}+\gamma_{\mathrm{NV}}$ at $T=T_{\mathrm{tr}}$ and 


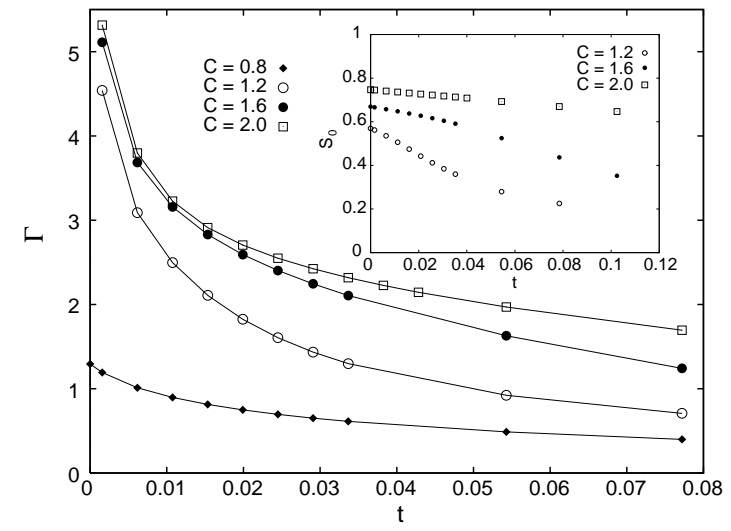

Fig. 3.

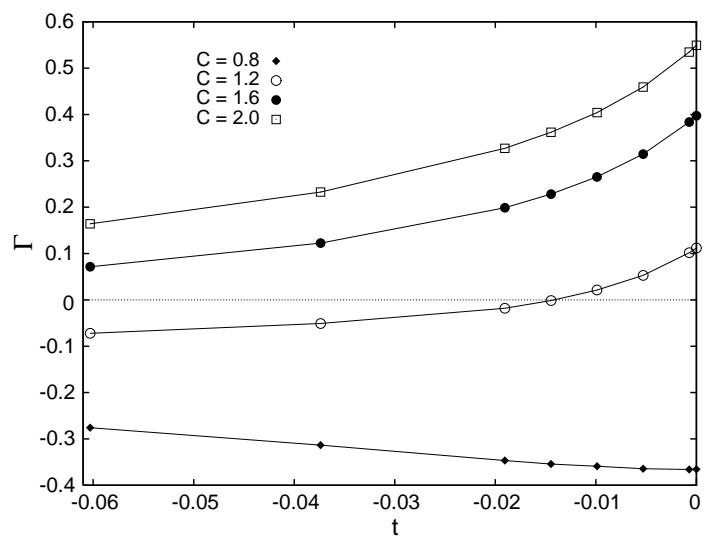

Fig. 4.

Fig. 3. - Nematic coverage, $\Gamma$, at the isotropic liquid-vapour interface as a function of the scaled temperature, $t$, for the systems of fig. 1 . In the inset, the surface order parameter, $S_{0}$, is plotted as a function of $t$ (see text for details). $\Gamma$ is in units of $\sigma$.

Fig. 4. - Nematic coverage, $\Gamma$, at the nematic-vapour interface as a function of the scaled temperature, $t$, for the systems of fig. 1 . $\Gamma$ increases as $|t|$ decreases towards the triple-point temperature for systems with $C \geq 1.0 . \Gamma$ is in units of $\sigma$.

the nematic coverage diverges as $t \rightarrow 0^{+}$, while it remains finite otherwise $(C \leq 0.8)$. Since the model interactions decay as $r^{-6}$, the asymptotic behaviour of $\Gamma$ is $\Gamma \propto t^{-\frac{1}{3}}$ [5]. Within the range of temperatures used in the experiments $\left(t \geq 1.3 \times 10^{-3}\right)$ we obtain this asymptotic behaviour for systems with $C=1.8,2.0$, only. A good fit to our data for these systems is $\ln \Gamma=b-0.29 \ln t$ which is the predicted power law within numerical errors. For systems with $C$ in the range 1.0-1.6, however, we find that the best fit to our data is $\Gamma \propto \ln t$. This logarithmic growth, is due to the exponential decay of the order parameter profiles. Within the experimental temperature range, we observe a crossover between the logarithmic and power law behaviours [11] for the systems with the largest values of $C$, i.e. the complete-wetting scaling region increases with $C$, as expected. This crossover takes place at $t \approx 10 \times 10^{-3}$ for $C=2.0$ and at $t \approx 5 \times 10^{-3}$ for $C=1.8$.

There is a controversy regarding the roles played by the excluded-volume (hard-core interactions) and the long-range anisotropic interactions (attractive on average) as the driving mechanism for the long-range orientational order of the bulk nematic phase. A logarithmic growth of nematic-wetting layers at LC interfaces has been reported by several authors [4], [12]. This fact could be used to conclude that the hard-core (short-range) interactions are the driving mechanism of nematic-wetting behaviour which would suggest, in turn, that these interactions play the main role as the driving mechanism of the bulk phase transition. Our results, however, indicate that this interpretation should be taken with care, since even when long-range forces are responsible for the bulk nematic order a logarithmic growth of nematic-wetting layers can be found, in the range of temperatures accessible to experiments. We note, in passing, that the asymptotic behaviour reported for the $n \mathrm{CBs}[4]$ cannot be the true asymptotic behaviour for these systems. It is known that the $n$ CB molecules are tilted at the NI interface [13], while they are perpendicular to the NV interface [9], [14], [15]. If the nematic layer is sufficiently thick (larger than $l_{0}$, with $10^{-5} \mathrm{~cm} \leq l_{0} \leq 10^{-3} \mathrm{~cm}$ ), the director will distort over the surface layer in order to yield the equilibrium orientations at both the NI and NV interfaces. Under these conditions, the nematic coverage has been shown to diverge as $\Gamma \propto t^{-\frac{1}{2}}$ [11]. The largest 


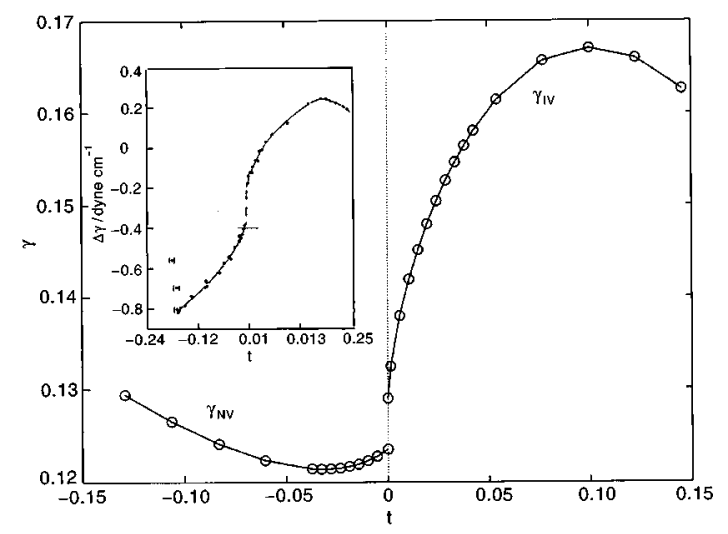

Fig. 5. - Interfacial tension, $\gamma$, of the liquid-vapour interface, for a system with $C=2.0$, as a function of the scaled temperature, $t$, close to the triple point, $t=0$. The tension increases with temperature both below and above the triple-point temperature (see text for details). $\gamma$ is in units of $\epsilon / \sigma^{2}$. The experimental results for $8 \mathrm{CB}[9]$ are shown in the inset.

layer thickness reported by Kasten and Strobl is $l \approx 200-250 \AA$ [4], which is too small to allow for this type of distorsion. Consequently, the clarification of the mechanisms which drive orientational-wetting phenomena requires further experiments.

We have also studied the nematic coverage at the $\mathrm{N}$ free surface when $t \rightarrow 0^{-}$. In fig. 4 we present our results. In systems exhibiting partial wetting $(C=0.8)$ no surface excess order develops at the NV interface as reported by Kasten and Strobl for 5CB [4]. In systems where wetting by $\mathrm{N}$ occurs $(C=1.0-2.0)$ the results of fig. 4 clearly indicate that the interfacial order increases as $T$ approaches $T_{\mathrm{tr}}$ from below. The general behaviour predicted by the theory closely resembles the experimental results.

Since Kasten and Strobl [4] have reported partial wetting at the IV interface of 5CB, a value $C<0.847$ is expected to describe this nematogen and we have taken $C=0.8$. As $n$ increases, the coupling between the orientational and translational degrees of freedom also increases and we will show, in the following, that $C=2.0$ yields a qualitative description of the surface properties of $8 \mathrm{CB}$.

We have chosen the maximum of the orientational order parameter profiles as the surface order parameter at the IV interface. When $C=2.0$ the surface order parameter is nearly independent of the temperature (see inset of fig. 3). The results reported by Kasten and Strobl [4] indicate that the surface order parameter of $8 \mathrm{CB}$ does not vary significantly as a function of $T$ and thus it is reasonable to take $C=2.0$ to model this nematogen.

Finally, several experiments [9], [16] reported an anomalous behaviour (positive values of $\mathrm{d} \gamma / \mathrm{d} T$ ) of the surface tension of nematogens above and below the triple-point temperature. One of these studies was carried out by Gannon and Faber [9] who measured the surface tension of $5 \mathrm{CB}$ and $8 \mathrm{CB}$ at temperatures close to $T_{\mathrm{tr}}$. Their results indicate that for these systems $\gamma$ increases with temperature in a range of temperatures just above $T_{\operatorname{tr}}$ (IV interface) and below $T_{\mathrm{tr}}$ (NV interface). At $T_{\mathrm{tr}}$ the surface tension exhibits a discontinuity $\left(\Delta \gamma=\gamma_{\mathrm{IV}}-\gamma_{\mathrm{NV}}>0\right)$ reflecting the first-order character of the bulk transition. At equilibrium, $|\Delta \gamma| \leq \gamma_{\mathrm{NI}}$, with the equality holding in the complete-wetting regime [5].

The positive value of $\mathrm{d} \gamma / \mathrm{d} T$ in the isotropic phase, just above the NI transition, was obtained previously using this model, and was shown to occur in systems where the IV interface is completely wet by $\mathrm{N}$ [7]. This behaviour is found in systems with $C>0.847$. The range over 
which $\gamma$ increases with $T$ becomes wider for larger values of $C$. For moderately large values of $C(C \leq 1.6) \gamma_{\mathrm{NV}}$ is found to decrease monotonically with temperature. When larger values of $C$ are considered, however, $\mathrm{d} \gamma / \mathrm{d} T$ becomes positive in a range of temperatures just below $T_{\mathrm{tr}}$ (see fig. 5). A comparison of our results and those of [9] illustrates how well our simple model captures the behaviour of the free-surface tension of $8 \mathrm{CB}$.

The anomalous behaviour of $\gamma$ below $T_{\mathrm{tr}}$ appears to be related to the anomalous surface order which develops at the NV interface for these systems. Although Gannon and Faber invoked surface-induced smectic order to explain their results [9], a detailed calculation was lacking. We note, however, that the mechanism proposed here is not related to wetting of the NV by the smectic phase, since the excess orientational order increases as the NI (and not the nematic-smectic) transition is approached.

We would like to stress that the simplicity of the model is a crucial ingredient of our findings. The generality (and applicability) of our results to real systems stems from the fact that we did not have to fine-tune a variety of intermolecular parameters to obtain the experimentally observed trends. Although a complete description of the interfacial properties (including the tilt angle at both interfaces) of the $n \mathrm{CB}$ series requires the inclusion of more terms in the intermolecular potential, as we have shown elsewhere [8], the main features of the surface-induced order and the wetting behaviour at the free surface of these systems is described accurately by the simple model presented here, with one notable exception, namely $5 \mathrm{CB}$. Indeed, if partial wetting occurs in $5 \mathrm{CB}$ as claimed by Kasten and Strobl [4], the behaviour of $\gamma$ found experimentally by Gannon and Faber [9] cannot be explained within our model. However, Beaglehole [12] has reported experimental evidence of complete wetting at the IV interface of $5 \mathrm{CB}$ indicating that the wetting behaviour of this nematogen depends on the sample used. Consequently new measurements of the surface tension and excess surface order for a given sample of $5 \mathrm{CB}$ are required, in order to conclude if the surface behaviour of this system is beyond the theoretical description based on the simple model given by eq. (1).

This work was supported in part by DGICYT of Spain through project PB91-0602 and by JNICT of Portugal through project PBIC/C/CEN/1844/92.

\section{REFERENCES}

[1] Jerome B., Rep. Prog. Phys., 54 (1991) 391.

[2] De Gennes P.-G., Mol. Cryst. Liq. Cryst., 12 (1971) 193.

[3] Telo da Gama M. M., Phys. Rev. Lett., 59 (1987) 154.

[4] Kasten H. and Strobl G., J. Chem. Phys., 103 (1995) 6768.

[5] Telo da Gama M. M., Mol. Phys., 52 (1984) 585.

[6] Thurtell J. M., Telo da Gama M. M. and Gubbins K. E., Mol. Phys., 54 (1985) 321.

[7] Tuipto-Margo B., Sen A. K., Mederos L. and Sullivan D. E., Mol. Phys., 67 (1989) 601.

[8] Martín del Río E., Telo da Gama M. M., De Miguel E. and Rull L. F., Phys. Rev. E, 52 (1995) 5028.

[9] Gannon M. G. J. and Faber T. E., Philos. Mag. A, 37 (1978) 117.

[10] Mederos L. and Sullivan D. E., Phys. Rev. A, 39 (1989) 854.

[11] Sullivan D. E. and Lipowsky R., Can. J. Chem., 66 (1988) 553.

[12] Beaglehole D., Mol. Cryst. Liq. Cryst., 89 (1982) 319.

[13] Faetti S. and Palleschi V., Phys. Rev. A, 30 (1984) 3241.

[14] Proust J. E. and Ter-Minassian-Saraga L., J. Phys. (Paris), 40 (1979) C3-490.

[15] Yokoyama H., Kobayashi S. and Kamei H., Mol. Cryst. Liq. Cryst., 99 (1983) 39.

[16] Krishnaswamy S. and Shashidhar R., Mol. Cryst. Liq. Cryst., 35 (1976) 253. 